\title{
Movilizando conocimientos a través del programa RNAO: Un movimiento social
}

\author{
Mobilizing Knowledge through the RNAO Program: A Social \\ Movement
}

\section{Mobilizando conhecimentos através do programa RNAO: um movimento social}

\author{
"Si no progresamos en nuestra Enfermería cada año, cada mes, \\ cada semana - toma mi palabra, estamos retrocediendo".
}

(Florence Nightingale)

Gracias Dra. Jaimes-Valencia, editora de la revista MedUNAB, por invitarme a escribir la editorial de este volumen, y me disculpo con los lectores por haber retrasado su difusión. El motivo de este retraso es una falencia conocida por la gran mayoría de ustedes: sobrecarga de trabajo - con todo importante, todo urgente y afortunadamente todo también ¡maravilloso!.

Se trata de que además de seguir luchando junto a nuestras 41,000 enfermeras miembros de la asociación - por la justicia social, la equidad, y las políticas sanitarias saludables he estado viajando por el mundo compartiendo el programa de guías de buenas prácticas de la Registered Nurses' Association of Ontario (RNAO). De hecho, escribo este editorial desde la ciudad de Chengdu en Sichuan, China.

Y, hay algo más - que es importante contarles: En Abril se lanza el libro Transforming nursing through knowledge: Best practices for guideline development, implementation science, and evaluation (Transformado a la Enfermería a Través del Conocimiento: Mejores Prácticas en Desarrollo de Guías, Ciencia de Implementación y Evaluación) (1). Publicado por SIGMA (anteriormente conocido como Sigma Theta Thao or STTI). Este libro es co-editado por Doris Grinspun e Irmajean Bajnok y contiene capítulos de muchas de las líderes de enfermería que han ya publicado en la revista MedUNAB, inclusive aquellas que están como autoras en esta edición. Por ahora el libro está disponible solo en Ingles, pero anticipamos que de aquí a cuatro meses estará también en español y chino. Para detalles visiten nuestra página web: www.RNAO.ca o me mandan un email a:DGrinspun@RNAO.CA

Vamos ahora de grano a este gran número de MedUNAB. Encontrarán aquí un artículo original interesantísimo del hospital FOSCAL en Bucaramanga, Colombia. En este Esparza-Bohórquez, Granados-Oliveros, SerranoSanmiguel y Peñaloza-Jaimes, describen sus experiencias en el cuidado de enfermería aplicando la guía de buenas prácticas de RNAO sobre prevención de caídas y lesiones derivadas en adultos mayores hospitalizados (2). También comparten los excelentes resultados alcanzados, especialmente el efecto positivo en la calidad de los cuidados.

En las páginas 207 a 214 de este mismo volumen, BelmarValdebenito, Díaz-Fluhmann, Abad y Sepulveda-Schaaf de Clínica las Condes, en Santiago de Chile, reflexionan sobre su gran experiencia en capacitación de Champions de la evidencia a través de E-learnings. Les recuerdo que Champions son aquellas enfermeras $\mathrm{u}$ otros profesionales de salud entrenados por RNAO para avanzar la adopción de evidencia en la práctica del día a día. El artículo de Belmar y colegas provee conclusiones importantes sobre el beneficio de programas E-learnings. 
Por otro lado, Bonilla-Marciales, Jaimes-Valencia, SerranoGómez, Arenas-Luna, Padilla-García, y Criado-Morales, de la Universidad Autónoma de Bucaramanga (UNAB), nos dan sus reflexiones sobre la integración de guías de buena práctica RNAO en la malla curricular. Su artículo titulado Implementación de la guía de valoración y manejo del dolor, de la Registered Nurses' Association of Ontario (RNAO) en el Programa de Enfermería de la Universidad Autónoma de Bucaramanga, comparte su experiencias y motivación para unirse al programa BPSO; esta última como un deseo de innovar con un currículo globalizado que responda a las necesidades del país y del mundo. Es así como eligen implementar a nivel curricular la guía de buenas prácticas clínicas de valoración y manejo de dolor (3) basadas en la evidencia científica. Su meta claramente es mejorar la calidad del cuidado de enfermería ofrecido por los profesionales en formación. En sus reflexiones a partir de la página 148, las autoras presentan la experiencia de implementación e incluyen la metodología escogida y como marco de referencia el Proyecto Educativo Institucional (PEI) de la UNAB. También captan de modo excelente el levantamiento de información que permitiera identificar los vacíos en los contenidos curriculares respecto al uso de recomendaciones de práctica clínica para la valoración y manejo del dolor en el currículo del programa de enfermería. Concluyen dándonos los próximos pasos que incluyen una transversalización de los contenidos relacionados con las buenas prácticas en las diferentes asignaturas del componente básico, profesional y específico.

Bonner, Hurley y Dabars, nos envían desde Australia sus experiencias como BPSO Host (anfitrión) llevando el programa BPSO en cinco instituciones en Adelaide (Australia del Sur). Ellos describen el proceso que llevaron - y aún siguen - para expandir el programa de guías RNAO a través de sus BPSOs. Un gran aporte de este trabajo es su análisis económico que demuestra el aporte positivo de reformas clínicas - como lo es el uso sistematizado de guías basadas en evidencia - en mejores resultados de salud para pacientes y menores gastos para organismos sanitarios. Les cuento con orgullos que junto a mi colega la Dra. Valerie Grdiza - Directora del Centro IABPG de la RNAO, tuvimos el placer en febrero del presente año de efectuar la auditoria. Ambas quedamos tremendamente impresionadas con los resultados alcanzados por cada una de las instituciones lideradas por Australian Nursing and Midwifery Federation (AMNF). La AMNF es el gremio más grande de enfermeras, matronas y asistentes de enfermería en toda Australia.

Sin quedarme yo atrás, les comparto en este número mis reflexiones en un artículo titulado Modelo de Enfermería para optimizar sistemas de salud. En este, incorporo ideas y herramientas que he ido desarrollando a través de los años y que han ayudado a ordenar nuestros pensamientos y espacios de acción. La discusión se centra en los desafíos claves de salud y atención sanitaria que enfrentan diversos países del mundo, y las amenazas y oportunidades que estos presentan para Enfermería. Se enfatiza la necesidad del uso de evidencia como una herramienta central pero no la única para optimizar los resultados de salud de los ciudadanos.

Estos artículos, y sus autores, comparten una noble idea: el deseo ferviente de transformar la enfermería a través del conocimiento, con el objeto de optimizar los cuidados de enfermería. Todos estos autores son grandes líderes de enfermería que, a través de arduo trabajo y dedicación increíble, están revolucionando nuestra profesión y con esto están incrementando nuestra contribución a nuestros sistemas de salud en forma local y global. A ellos, y a todos los líderes y Champions de las 700 instituciones de salud y académicas en el mundo entero les doy las gracias por su liderazgo inspirador, su capacidad de compartir y sus relaciones humanas exquisitas. Juntos, hemos construido un movimiento que ya tiene una identidad colectiva. Sobre este último punto escribiré en una edición futura de la revista MedUNAB.

Doris Grinspun, RN, MSN, PhD, LLD(hon), Dr(hc), O.ONT. Gerente General

Asociación de Enfermeras Registradas de Ontario

\section{Referencias}

1. Grinspun D, Bajnok I. Transforming nursing through knowledge: Best practices for guideline development, implementation science, and evaluation. Indianapolis, IN, USA: Sigma Theta Thau Internacional Honor Society or Nursing.

2. Registerd Nurses'Association of Ontario. Prevention of falls and fall injuries in the older adult (Revised). Toronto; 2012. Disponible en: http://rnao.ca/sites/rnaoca/files/Prevention_of_Falls_and_Fall_Injuries_in_the_ Older_Adult.pdf

3. Registered Nurses' Association of Ontario. Assessment and Management of Pain. 3rd ed. Toronto; 2013. Disponible en: http://rnao.ca/sites/rnaoca/files/AssessAndManagementOfPain_15_WEB_FINAL_DEC_2.pdf 